\title{
Functional analysis of the ABA-responsive protein family in ABA and stress signal transduction in Arabidopsis
}

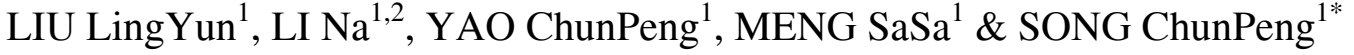 \\ ${ }^{1}$ State Key Laboratory of Cotton Biology, Laboratory of Plant Stress Biology, Department of Biology, Henan University, Kaifeng 475004, China; \\ ${ }^{2}$ Zhengzhou Shuqing Medical College, Faculty of Basic Medicine, Zhengzhou 450000, China
}

Received January 28, 2013; accepted April 12, 2013; published online July 25, 2013

\begin{abstract}
The phytohormone abscisic acid (ABA) plays an important role in plant growth and development, for example in seed dormancy and germination, as well as in plant responses to environmental stresses, such as drought and high salinity. Previous studies have shown that ABA regulates the expression of genes with an ABA-responsive element (ABRE) and their corresponding physiological responses. Bioinformatics analysis identified a GRAM domain-containing gene family that has a multiple ABRE cis-element, which was termed the ABA-responsive protein (ABR) family. To analyze the function of the ABR family, we identified homozygous T-DNA insertion mutants and constructed $a b r 1,2,3$ double mutants and triple mutant. The $a b r l, a b r 2$ and $a b r 3$ single mutants showed a normal phenotype; however, the germination of seeds of the double mutants and triple mutant were insensitive to ABA, $\mathrm{NaCl}$, mannitol and glucose. ABR1-GFP was distributed as a punctate structure in the cytosol and may be localized in the endomembrane system. The ABR2-GFP and ABR3-GFP proteins localized in the cytoplasm. In addition, ABR1, $A B R 2$ and $A B R 3$ were expressed in various tissues, and could be induced by several abiotic stresses, especially by ABA. The expressions of these genes were significantly suppressed in aba2, abil and abi2 null mutants. These results suggested that the ABR family may act downstream of $A B I 1$ and $A B I 2$ in the ABA signal transduction process in plants.
\end{abstract}

ABA-responsive protein, GRAM domain, ABA, germination, Arabidopsis thaliana

Citation: $\quad$ Liu L Y, Li N, Yao C P, et al. Functional analysis of the ABA-responsive protein family in ABA and stress signal transduction in Arabidopsis. Chin Sci Bull, 2013, 58: 3721-3730, doi: 10.1007/s11434-013-5941-9

The phytohormone ABA plays a key role in regulating different aspects of plant growth and development, as well as the plant response to biotic and abiotic stress [1,2]. Therefore, it is of great interest to reveal the molecular mechanism of the function of $\mathrm{ABA}$ in signal transduction. Recent advances in ABA signal transduction research include the discovery of ABA receptors and their associated molecular mechanisms. This research showed that PYRABACTION RESISTANCE (PYR) and REGULATORY COMPONENT OF ABA RECEPTOR (RCAR) are ABA receptors that are located in the vertex of the ABA signal transduction pathway to negatively regulate $\mathrm{ABA}$ signaling by inhibiting the activity of protein phosphatases (PP2Cs). ABA activates the plant stress response through binding to soluble PYR1/PYL/ RCAR receptors. Quadruple mutants of the ABA receptor

*Corresponding author (email: songcp@henu.edu.cn) encoding genes exhibited seed germination and root growth that was insensitive to ABA-regulation, as was expression of ABA-responsive genes. The PYR and PYL receptors recognize intracellular $\mathrm{ABA}$ and bind with the downstream PP2Cs such as ABI1 and ABI2. The resultant ternary complex activates sucrose non-fermenting 1-related subfamily2 protein kinase (SnRK2), which plays a key role in ABA signaling regulation, including response to $\mathrm{ABA}$ and regulation of stomatal opening [3-5]. Some genes induced by ABA during seed development of Arabidopsis have been cloned from Arabidopsis mutants, such as ABI3, ABI4, ABI5, LEC1, LEC2, FUS, MARDI and CIPK3. ABI3/VP1 protein is a transcription factor that is involved in the accumulation of reserves, the acquisition of seed desiccation tolerance and the induction of seed dormancy [6-9]. The ABI5-related bZIP transcription factor binds with the ABRE cis-element to activate ABA- 
mediated gene transcription in seeds. ABI5 is a critical target site in conserved ABA signal transduction [10]; at least seven genes of the 13 genes regulated by $A B 15$ related bZ1P transcription factors are expressed during seed development, and the expressions of these genes are antagonized, which suggests that they promote the ABA response with a specific function [11]. The interaction between $\mathrm{ABI} 3$ and $\mathrm{ABI} 5$ activates gene transcription process mediated by the ABRE and RY cis-elements. Thus, the ABREs may represent a crossover point among ABA signaling networks [12]. Although the basic framework of ABA signal transduction has been established, the detailed molecular mechanisms remain to be discovered.

To identify ABA-associated antigen protein analogs and new components in ABA signal transduction, an ABA antibody was used to screen a barley cDNA expression library. A cDNA (aba45) was identified that was induced by ABA in barley, whose expression products could be recognized by the anti-ABA monoclonal antibody [13]. Two aba45 homologs, which were named ABA-responsive genes (ABRs), were obtained by forming analog screening in the yeast two-hybrid system [14]. The ABRs not only contained ABRE cis-elements in their promoter regions but also contained a GRAM (glucosyltransferases, Rab-like GTPase activators and myotubularins) domain in the protein. The GRAM domain is a class of intracellular protein and lipid binding signal domain, which comprises about 70 amino acids and is frequently associated with the $\mathrm{PH}$ (the Pleckstrin Homology Domain), TBC (domain in Tre-2, BUB2p and $\mathrm{Cdc} 16 \mathrm{p}$ ), and $\mathrm{C} 2$ (protein kinase $\mathrm{C}$ conserved region 2) domains in proteins, and this domain is involved in membrane-bound process [15-19]. The Arabidopsis genome contains 13 genes, which encode ABRs containing GRAM domains. The proteins encoded by the At1g03370, At3g59660, and At5g50170 genes have a C2 domain and a GRAM domain; the remaining 10 proteins contain only a GRAM domain [20]. Although some GRAM domain proteins have been identified in Arabidopsis and rice, the molecular details of the involvement of these proteins in the response to $\mathrm{ABA}$ remains unclear.

In this study, we obtained three single-gene homozygous mutants (abrl, abr2 and $a b r 3)$ of the ABR gene family, as well as the double mutants ( $a b r 1 / 2, a b r 1 / 3$ and $a b r 2 / 3)$ and a triple mutant $a b r 1 / 2 / 3$. Assays of seed germination and seedling growth under stress conditions showed that $A B R 1$, $A B R 2$ and $A B R 3$ are involved in ABA-mediated inhibition of seed germination and may act downstream of $A B I 1$ and $A B I 2$ in the ABA signaling pathway.

\section{Material and methods}

\subsection{Plant materials and growth conditions}

Arabidopsis thaliana ecotype Columbia was used in this study. Seeds were sterilized and kept for $3 \mathrm{~d}$ at $4{ }^{\circ} \mathrm{C}$ in the dark to break dormancy. The seeds were then sown on MS medium containing $0.6 \%$ agar and incubated at $18-22^{\circ} \mathrm{C}$ with a 16-h-light/8-h-dark photoperiod and $70 \%$ relative humidity $(\mathrm{RH})$. Seedlings were transferred to soil at 7-10 d after germination and placed in a growth chamber at $22 \pm 2^{\circ} \mathrm{C}$ under a 16-h-light/8-h-dark photoperiod and 70\% RH, and watered every other day for normal growth conditions.

\subsection{Isolation of $a b r 1, a b r 2, a b r 3$ mutants}

The T-DNA insertion lines for ABRI (At4g01600) SALK 032205, ABR2 (At5g13200) SALK_013073 and ABR3 (At2g22475) SALK_145846 were ordered from the Arabidopsis Biological Resource Center (http://www.arabidopsis. org/abrc/). Homozygous mutant plants were screened by PCR using gene-specific primers: $a b r l$ : LP (5'-TTAACGGTCAAGACAACGACC-3'), RP (5'-ATCCTTTACATGATTTTGCGG-3'), abr2: LP (5'-AGTTGTTGGATGTTCGACAGG-3'), RP (5'-TCACCATAGATAGCATTCGGC-3'), abr3: LP (5'-TGAGAAGCTGATGCAATCATG3'), RP (5'-TGAATAGATGCTCCGGAACAG-3'), and vector left-border-specific primers LBal (5'-TGGTTCACGTAGTGGGCCATCG-3') and LBb1 (5'-GCGTGGACCGCTTGCTGCAACT-3'). The homozygous lines were backcrossed with wild-type (WT) plants, and the homozygous $F_{2}$ progeny were used for phenotype analysis.

\subsection{Plasmid constructs}

For the subcellular localization of $A B R 1, A B R 2, A B R 3$, gene-specific cDNA fragments of $A B R 1, A B R 2$, and $A B R 3$ were amplified by PCR. The following primer pairs were used to amplify the cDNA fragments for insertion into the pHBT-GFP plasmid: ABR1 forward primer 5'-CCCGTCGACATGCGTCCACAATACAAAG-3' and reverse primer 5'-TATCCCGGGCCCATGGCATGATTACAAC3', ABR2 forward primer 5'-CCCGTCGACATGACAGGATCACAAGAAG-3' and reverse primer 5'-TATCCCGGGCCACCAGACACAGAGCCGT-3', and ABR3 forward primer 5'-CCCGTCGACATGGAGCCGCCGAAGGGAG-3' and reverse primer 5'-TATCCCGGGCCCACCGACCTTAAAGCAC-3' (Sal I and Sma I restriction sites are underlined in forward and reverse primers, respectively).

\subsection{Plant protoplast transfection}

To observe the subcellular localization of the proteins of interest, protoplasts isolated from Arabidopsis leaves were transformed with plasmids $A B R 1-p H B T-G F P, A B R 2-p H B T$ $G F P$ and $A B R 3-p H B T-G F P$, as described previously [21].

\subsection{Confocal imaging for ABR1, ABR2, and ABR3 subcellular localization}

After incubation for $16-20 \mathrm{~h}$, the fluorescence of the proto- 
plasts was detected under a laser scanning confocal microscope (FV1000, Olympus). The protoplasts were excited at $488 \mathrm{~nm}$ and fluorescence was detected at 500-530 $\mathrm{nm}$ for GFP and at $650-750 \mathrm{~nm}$ for autofluorescence of chloroplasts. The mitochondrion-specific probe Mito-tracker was excited at $543 \mathrm{~nm}$ and detected at 550-650 nm, and transmitted fields were collected simultaneously for use in merged images. Magnification at $60 \times$ using an oil immersion lens was used to obtain high resolution images of cells.

\subsection{Semi-quantitative RT-PCR and real-time quantita- tive RT-PCR}

WT and mutant seedlings were grown in a chamber for 15 days at $22 \pm 2^{\circ} \mathrm{C}$ under a 16 -h-light/8-h-dark photoperiod and $70 \% \mathrm{RH}$, for normal growth conditions as controls. For stress treatments, plants were grown under normal growth conditions for $10 \mathrm{~d}$ and then stressed by $\mathrm{ddH}_{2} \mathrm{O}, 100 \mu \mathrm{mol} \mathrm{L} \mathrm{L}^{-1}$ ABA, $150 \mathrm{mmol} \mathrm{L}^{-1} \mathrm{NaCl}, 300 \mathrm{mmol} \mathrm{L}^{-1}$ mannitol, and dehydration treatment for $24 \mathrm{~h}$. Total RNA was isolated from 100-mg tissue samples using the TRIZOL reagent (Invitrogen, http://www.invitrogen.com/). First-strand cDNA was synthesized using Maloney murine leukemia virus reverse transcriptase (Promega, http://www.promega.com/). For semi-quantitative RT-PCR, the volume of each cDNA sample was adjusted to give the same signal strength for Actin2 after 20 cycles of PCR. The RT-PCR products were analyzed by electrophoresis on $1.5 \%$ agarose gels. SYBR Premix Ex Taq (TaKaRa) and a Stratagene MX3005P apparatus were used for real-time RT-PCR. The cycling conditions were as follows: $30 \mathrm{~s}$ at $95^{\circ} \mathrm{C}$; 40 cycles of $15 \mathrm{~s}$ at $95^{\circ} \mathrm{C}, 20 \mathrm{~s}$ at $56^{\circ} \mathrm{C}$, and $30 \mathrm{~s}$ at $72^{\circ} \mathrm{C}$. This was followed by a melting-curve program $\left(60 \mathrm{~s}\right.$ at $95^{\circ} \mathrm{C} ; 30 \mathrm{~s}$ at $55^{\circ} \mathrm{C} ; 30 \mathrm{~s}$ at $95^{\circ} \mathrm{C}$ ). Specific cDNAs were quantified using a standard curve based on known amounts of amplified target gene fragments. The mean value of three replicates was normalized against Actin2 as the internal control. Real-time quantitative PCR experiments were repeated three times.

\section{Results}

\subsection{Bioinformatics analysis of ABR family protein}

Bioinformatics analysis by the http://www.arabidopsis.org/, and http://www.ebi.ac.uk/Tools/pfa/iprscan/showed that ABR1 is a $25.91 \mathrm{kD}$ protein comprising 233 amino acids, ABR2 is a $30.07 \mathrm{kD}$ protein comprising 272 amino acids, and ABR3 is a $32.21 \mathrm{kD}$ protein comprising 299 amino acids; these proteins each have one GRAM domain (Figure 1 (a)-(c)). The GRAM domain is a structural characteristic of ABA-responsive protein [15], and these results suggest that $\mathrm{ABR} 1, \mathrm{ABR} 2$ and $\mathrm{ABR} 3$ are $\mathrm{ABA}$-responsive proteins that transfer the ABA signal and induce the corresponding ABA response in plants.

As ABA-responsive genes, the genes that encode pro- teins containing GRAM domain could have ABA-responsive ABRE cis-acting elements. The cis-acting elements of $A B R 1, A B R 2$, and $A B R 3$ genes were predicted by the website http://www.dna.affrc.go.jp/PLACE/. The prediction results showed that the ABRE cis-acting element ACGTG [22], which has been found in ABA response gene $A B I 3$, $R D 29 A, R D 29 B$, is present at +8 bp downstream of the initiation codon ATG in $A B R 1$ gene (Figure 1 (d)). In $A B R 2$, an ABRE cis-acting element, ACGTG, was found at positions -419 and -396 bp upstream of the ATG codon. Another ABRE cis-acting element, MACGYGB [23], was found at positions $-420,-397,-684$ and -129 bp (Figure 1 (e)). In ABR3, an ABRE cis-acting element, ACGTG, was detected at positions $-381,-808,-2471,-2397$ and -2349 bp upstream of the ATG codon. In addition, the ABRE cis-acting element MACGYGB was detected at positions $-2226,-1877,-403,-144,-2398,-2349,-2284$ bp. The ABRE cis-acting element YACGTGGC, which was found in the promoters of $A B F$ genes [24-27] was detected at position $-2400 \mathrm{bp}$, and the ABRE the cis-acting element RYACGTGGYR, which was found in the promoter of the dehydration-induced ABA-regulated gene RD22 [28-30], was detected at position -2401 bp (Figure 1 (f)). Thus, $A B R 1, A B R 2$ and $A B R 3$ have many ABRE cis-acting elements, which indicate that these genes might be involved in the response to ABA.

2.2 Deficiency of $A B R 1, A B R 2$ and $A B R 3$ results in ABA-insensitive seed germination and early seedling growth in Arabidopsis

To analyze the ABR family gene function in to response to ABA, we obtained gene deletion mutants from the Arabidopsis seeds Resource Center, and used them to construct double or triple mutant of the ABR genes. The T-DNA insertion deletion mutants SALK_032205 (abrl), SALK_ 013073 (abr2), and SALK_145846 (abr3) of ABRI (At4g01600), $A B R 2$ (At5g13200), and ABR3 (At2g22475 GEM) were identified. In these mutants, the T-DNA was inserted in the fourth exon of the $A B R 1$ gene, in the promoter region of the $A B R 2$ gene and in the second intron of the $A B R 3$ gene, respectively (Figure 2 (a)). The insertion was expected to eliminate or strongly reduce the transcription of $A B R 1, A B R 2$ and $A B R 3$, which was confirmed by reverse transcriptase-PCR (RT-PCR) analyses (Figure 2(b)). To better study the function of these genes, we constructed double mutants $a b r 1 / 2, a b r 2 / 3$ and $a b r 1 / 3$, and a triple mutant $(a b r 1 / 2 / 3)$. We also verified that these double and triple mutants were null mutants by RT-PCR (Figure 2(c) and (d)).

First, we observed the seed germination of $a b r 1, a b r 2$, and $a b r 3$ single mutants with different concentrations of ABA, and found no significant differences between the single mutants and the WT (Figure 2 (e)). We also monitored leaf temperature changes of the seedlings in normal and 
(a)

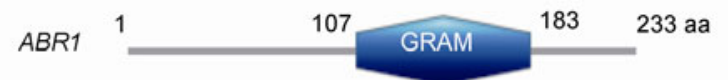

(b)

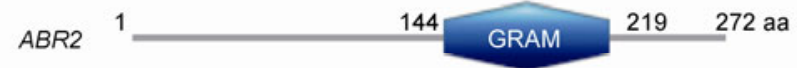

(c)

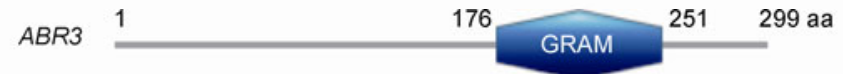

(d)

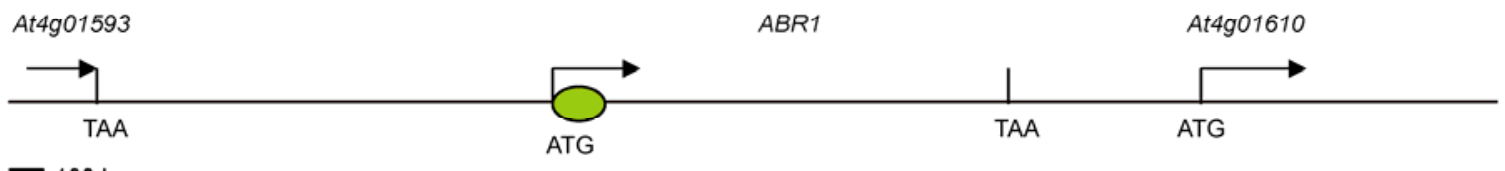

$-100 \mathrm{bp}$

At5g13190

$A B R 2$

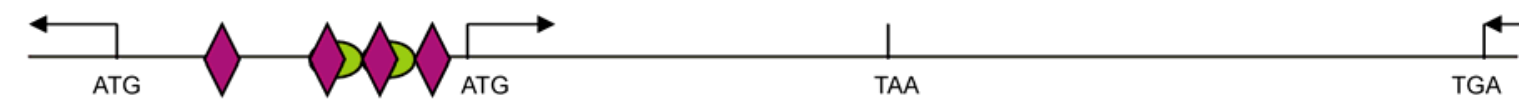

$-100 \mathrm{bp}$

(f)

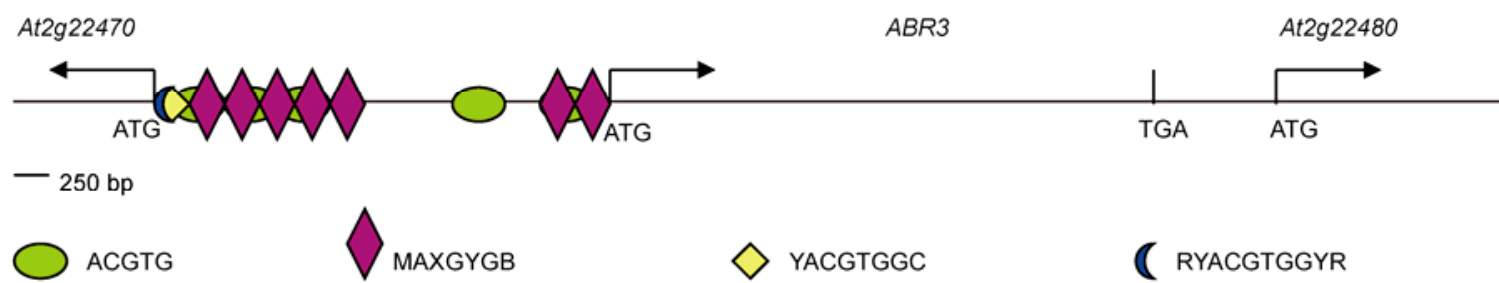

Figure 1 Protein structure of ABR1, ABR2, and ABR3, and the ABRE cis-acting element distribution in the corresponding genes. (a)-(c) Location of GRAM domains in the ABR1 ABR2, and ABR3 proteins, respectively. (d)-(f) Distribution of ABRE cis-acting elements in the $A B R 1, A B R 2, A B R 3$ genes, respectively.

drought conditions using a far-infrared imager. No significant differences were observed between the single mutants and the WT. Interestingly, the seed germination rate was consistent among the double mutants $a b r 1 / 2, a b r 1 / 3, a b r 2 / 3$, the triple mutant $a b r l / 2 / 3$ and the WT in MS medium, However, when the MS medium was supplemented with 0.5 , 1.0 and $1.5 \mu \mathrm{mol} \mathrm{L}{ }^{-1} \mathrm{ABA}$, the seed germination rate of the double mutants were generally higher than the WT, by approximately 18\%, 20\% and 22\%, respectively (Figure 2 (f)). In MS medium supplemented with $0.5 \mu \mathrm{mol} \mathrm{L}{ }^{-1} \mathrm{ABA}$, the seed germination rate of the $a b r 1 / 2 / 3$ triple mutant was higher than of the WT by approximately $24 \%$ (Figure 2 (g2) and $(\mathrm{h})$ ). Moreover, the seed germination rates showed no differences between the double mutants and the WT when subjected to other stresses. However, under $\mathrm{NaCl}$ (Figure 2 (g3) and (i)), mannitol (Figure 2 (g5) and (j)) and glucose (Figure $2(\mathrm{~g} 6)$ and $(\mathrm{k})$ ) treatments, seed germination rate of the triple mutant was higher than that of the WT. These results showed that the abiotic stress tolerance of the $a b r 1 / 2 / 3$ triple mutant was higher than that of the WT, and indicated that seed germination of the triple mutant was insensitive to ABA, $\mathrm{NaCl}$, mannitol and glucose. To further understand the phenotype of these mutants under stress conditions, we measured water loss of leaves and performed far-infrared imaging experiments. The results suggested that the rate of water loss from the leaves of each mutant and the WT were similar. The infrared imager monitoring results also showed that leaf temperature changes of the mutant and WT plants were very similar under drought stress or normal growth conditions (data not shown). These results illustrate that the $A B R 1, A B R 2$, and $A B R 3$ genes are indeed involved in the ABA-regulated abiotic stress response during seed germination process; however, they also suggest that $A B R 1, A B R 2$ and $A B R 3$ are functionally redundant in Arabidopsis.

\subsection{ABR1, ABR2 and ABR3 are localized in the endo- membrane system and the cytoplasm, respectively}

To further reveal regulation mechanism of $A B R 1, A B R 2$ and $A B R 3$ in response to $\mathrm{ABA}$, we determined the subcellular 

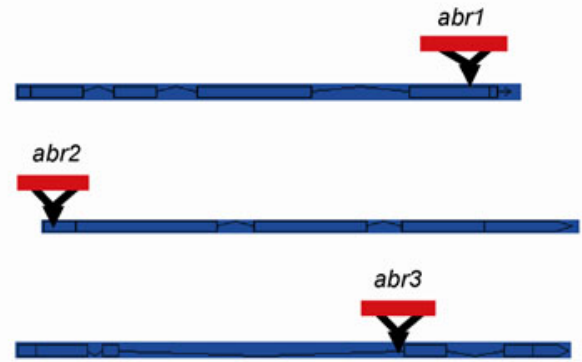

(d)

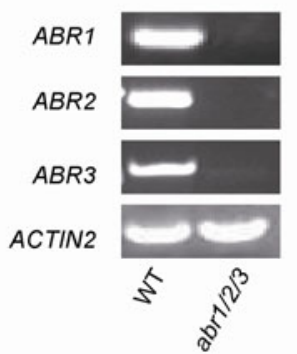

(e)

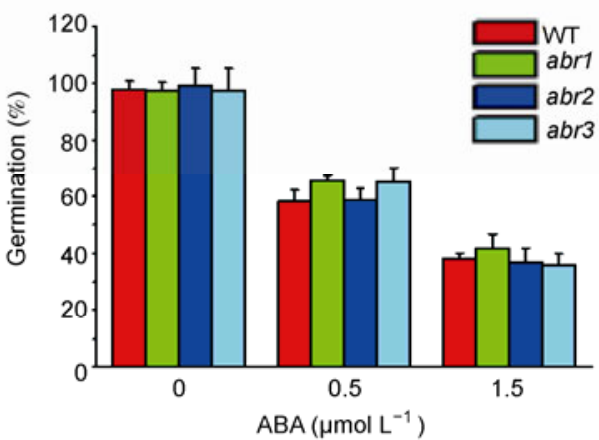

(g)

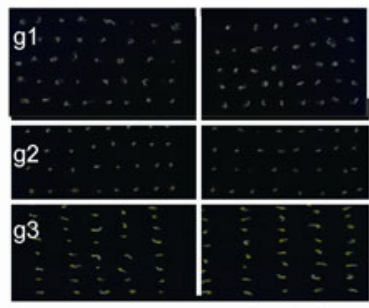

WT

$a b r 1 / 2 / 3$

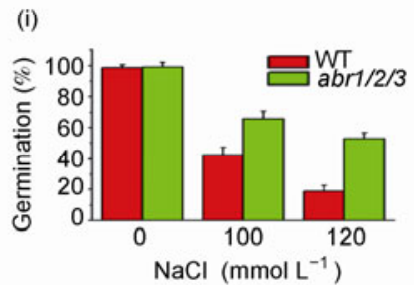

(b)

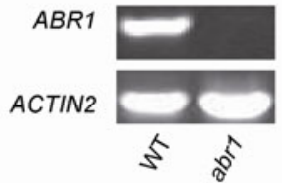

ABR2

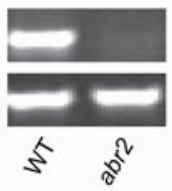

$A B R 3$

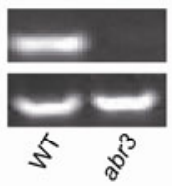

(c)
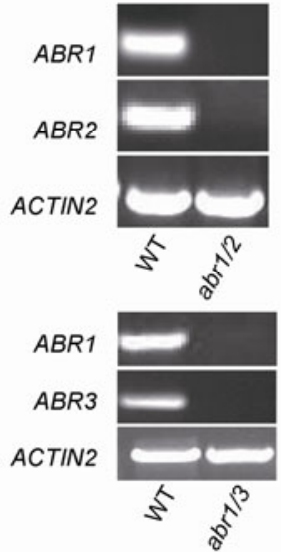

$A B R 2$

$A B R 3$

ACTIN2

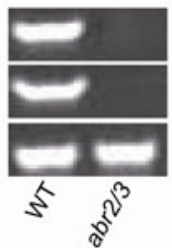

(f)

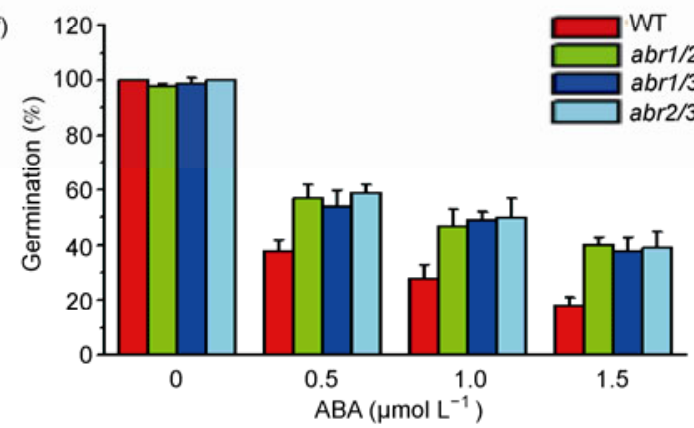

(h)
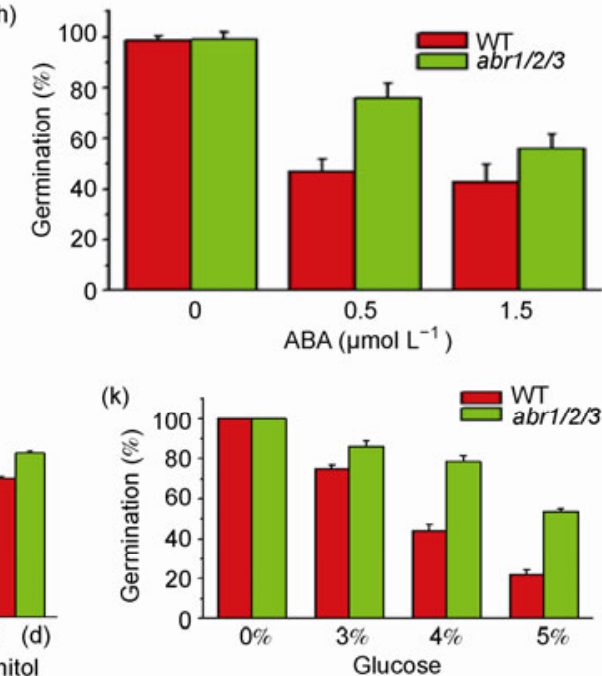

Figure 2 Homozygous $a b r 1$, abr2 and abr3 single mutants, double mutant and triple mutant: Identification and response to ABA, NaCl, mannitol and glucose. (a) Insertion position of the T-DNA in the $A B R 1, A B R 2$ and $A B R 3$ genes; (b)-(d) reverse transcription PCR analysis of $A B R 1, A B R 2, A B R 3$ expression. Actin2 served as the control; (e) and (f) Phenotype analysis of the WT and $a b r 1, a b r 2$, abr3 single mutants and double mutants grown in MS medium and MS medium supplemented with different concentrations of ABA, respectively; $(\mathrm{g}) \mathrm{g} 1-\mathrm{g} 3$, seed germination on $\mathrm{MS}$ with or without $0.5 \mu \mathrm{mol} \mathrm{L}^{-1} \mathrm{ABA}$, $100 \mathrm{mmol} \mathrm{L}^{-1} \mathrm{NaCl}$ on the fourth day, g4-g6, Seed germination on MS medium with or without $300 \mathrm{mmol} \mathrm{L}^{-1} \mathrm{~N}$ mannitol, 5\% glucose on the 10 th day; (h)-(k) Phenotype analysis of the WT and $a b r 1 / 2 / 3$ triple mutant in MS with or without different concentrations of ABA, NaCl, mannitol and glucose. About 100 seeds of each genotype were used, radicle appearance was used as the evaluation index, and values are means \pm SD of four samples. 
localization of ABR1, ABR2 and ABR3 by transient gene expression analysis in mesophyll protoplasts of Arabidopsis, using the ABR1-pHBT-GFP, ABR2-pHBT-GFP, and ABR3pHBT-GFP plasmids, separately. The results showed that the ABR1-GFP fusion protein is present in specific cellular compartments that showed a punctate distribution (Figure 3 (a1)). Subsequently, to determine whether the ABR1-GFP fusion protein is located in the mitochondria, the mitochondria-specific Mito-tracker Red probe was incubated with mesophyll protoplasts expressing ABR1-pHBT-GFP. The green fluorescence of the ABR1-GFP fusion protein partially co-localized with the red fluorescence of the Mito-tracker Red probe (Figure 3 (a1)-(a6)). Meanwhile, the green fluorescent protein was localized throughout the cells when the protoplasts were transformed with the pHBT-GFP vector control (Figure 3 (b1), (b2)). Similarly, the green fluorescent protein was localized throughout the cells when protoplasts were transformed with the ABR2-pHBT-GFP and ABR3-pHBT-GFP plasmids separately (Figure 3(c1)(c4) and (d1)-(d4)). These results indicated that ABR1 is localized in cytoplasmic non-mitochondrial organelles, which may be part of the endomembrane system. We spec- ulate that $\mathrm{ABR} 1$ is involved in $\mathrm{ABA}$ signal transduction through the binding of its GRAM domain to the endomembrane system. Both ABR2 and ABR3 are localized in the cytoplasm, and ABA receptor PYR1 is also located in the cytoplasm [5]; these results indicate that ABR2 and ABR3 participate in the ABA signaling pathway in cytoplasmic compartments.

\section{4 $A B R 1, A B R 2$ and $A B R 3$ are induced by different abiotic stresses, and expressed in various tissues}

To detect the tissue-specific expression of $A B R 1, A B R 2$ and $A B R 3$ in plants, we conducted detailed analyses of $A B R I$, $A B R 2$ and $A B R 3$ expression by real-time RT-PCR. RT-PCR analysis showed that $A B R 1, A B R 2$ and $A B R 3$ were transcribed in various organs, including the roots, stems, leaves, flowers, and siliques. The expression of $A B R 1$ was higher in various tissues than that of $A B R 3$, especially in leaves, which was similar to the expression pattern of the $A B R 2$ (Figure 4(a) and (b)). The expression of $A B R 3$ was relatively low in various tissues (Figure 4(c)). Differences in tissue-specific expression among $A B R 1, A B R 2$ and $A B R 3$ may
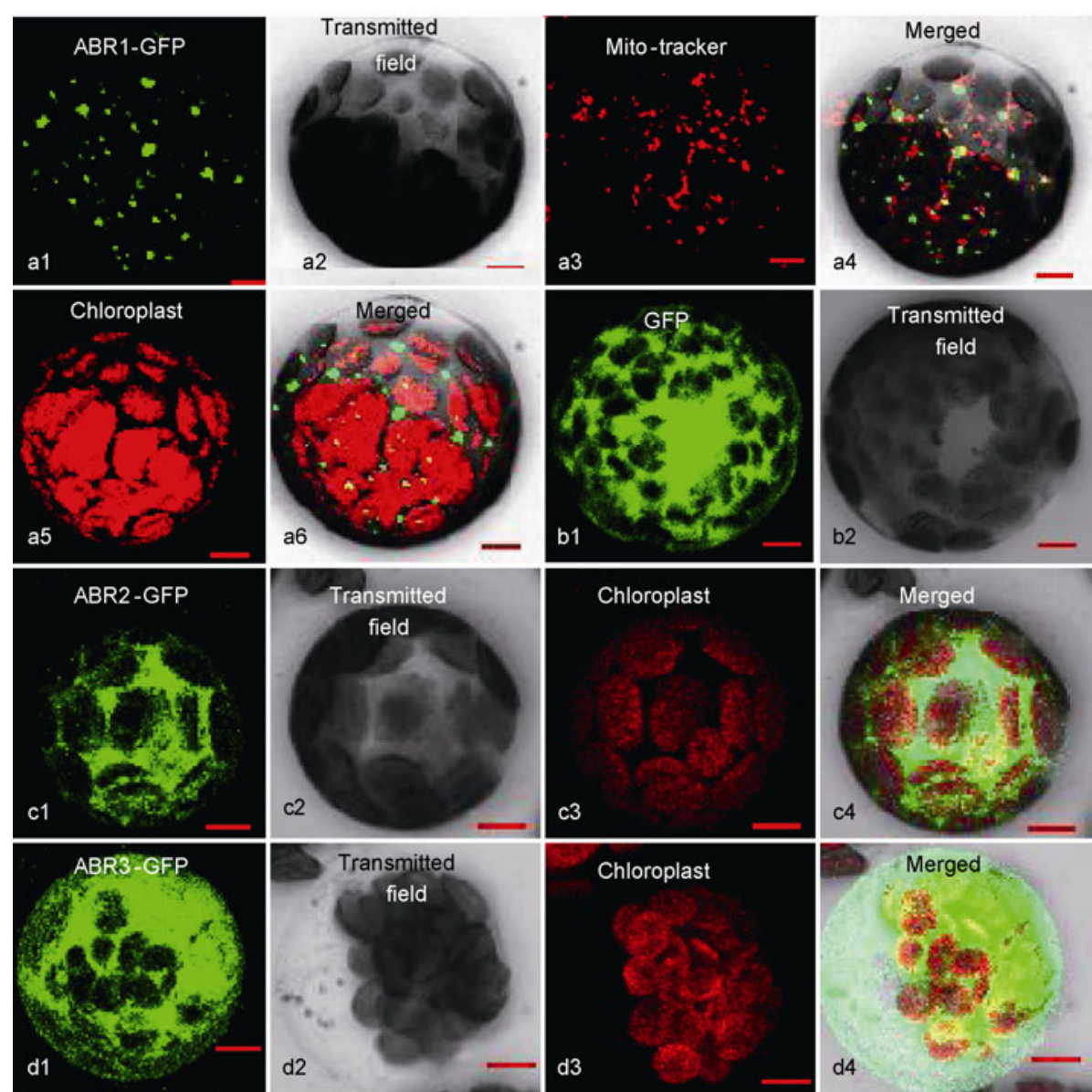

Figure 3 Subcellular localization of ABR1, ABR2 and ABR3 in protoplasts. (a1)-(a6) Green fluorescence of the ABR3-GFP fusion protein and red fluorescence of Mito-tracker are superimposed. (b1) and (b2) Green fluorescent protein alone was localized throughout cells when they were transformed with the pHBT-GFP vector control. (c1)-(c4) Green fluorescence of the ABR2-GFP fusion protein. (d1)-(d4) Green fluorescence of the ABR3-GFP fusion protein. Bar $=5 \mu \mathrm{m}$. 

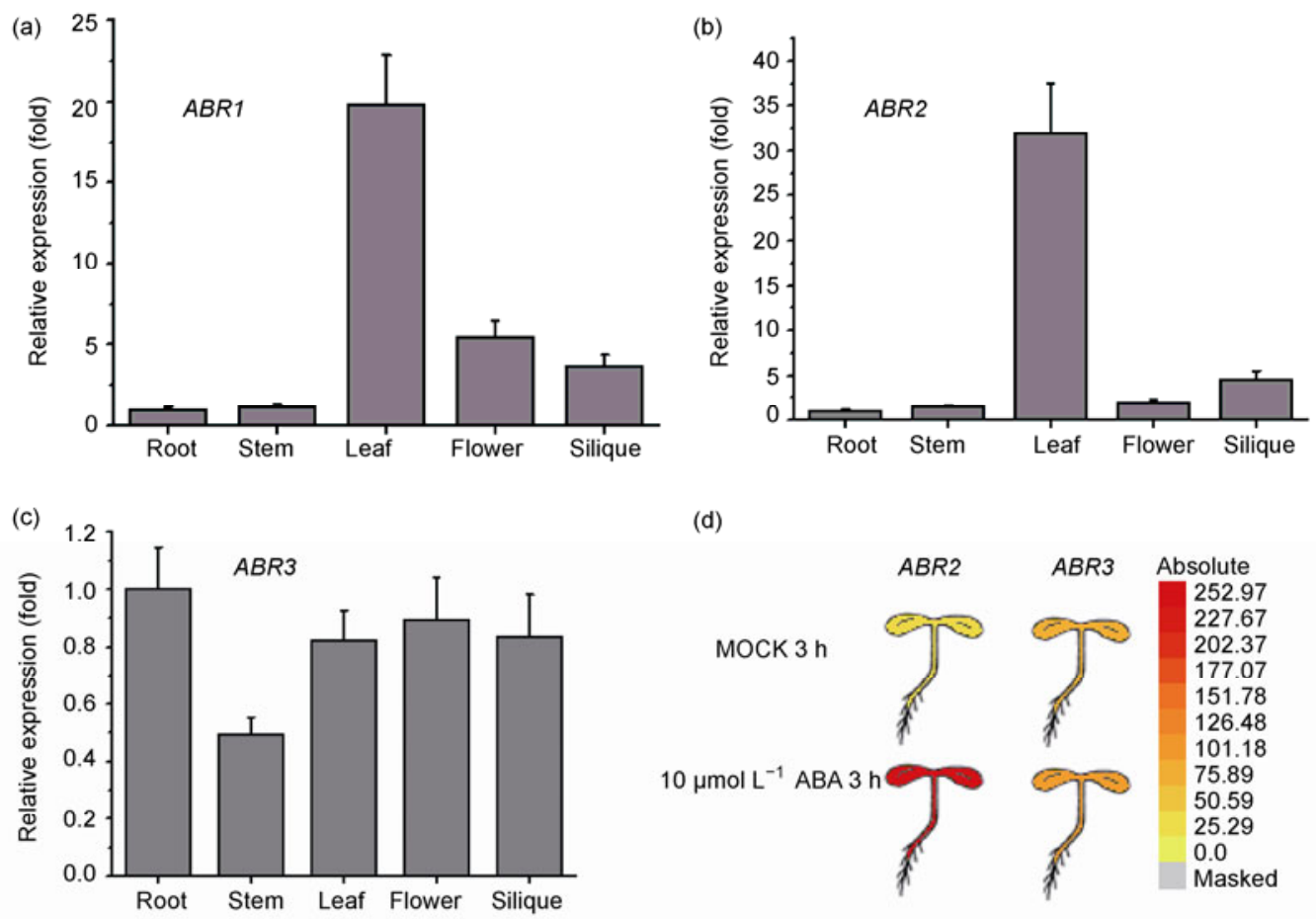

(d)

(e)
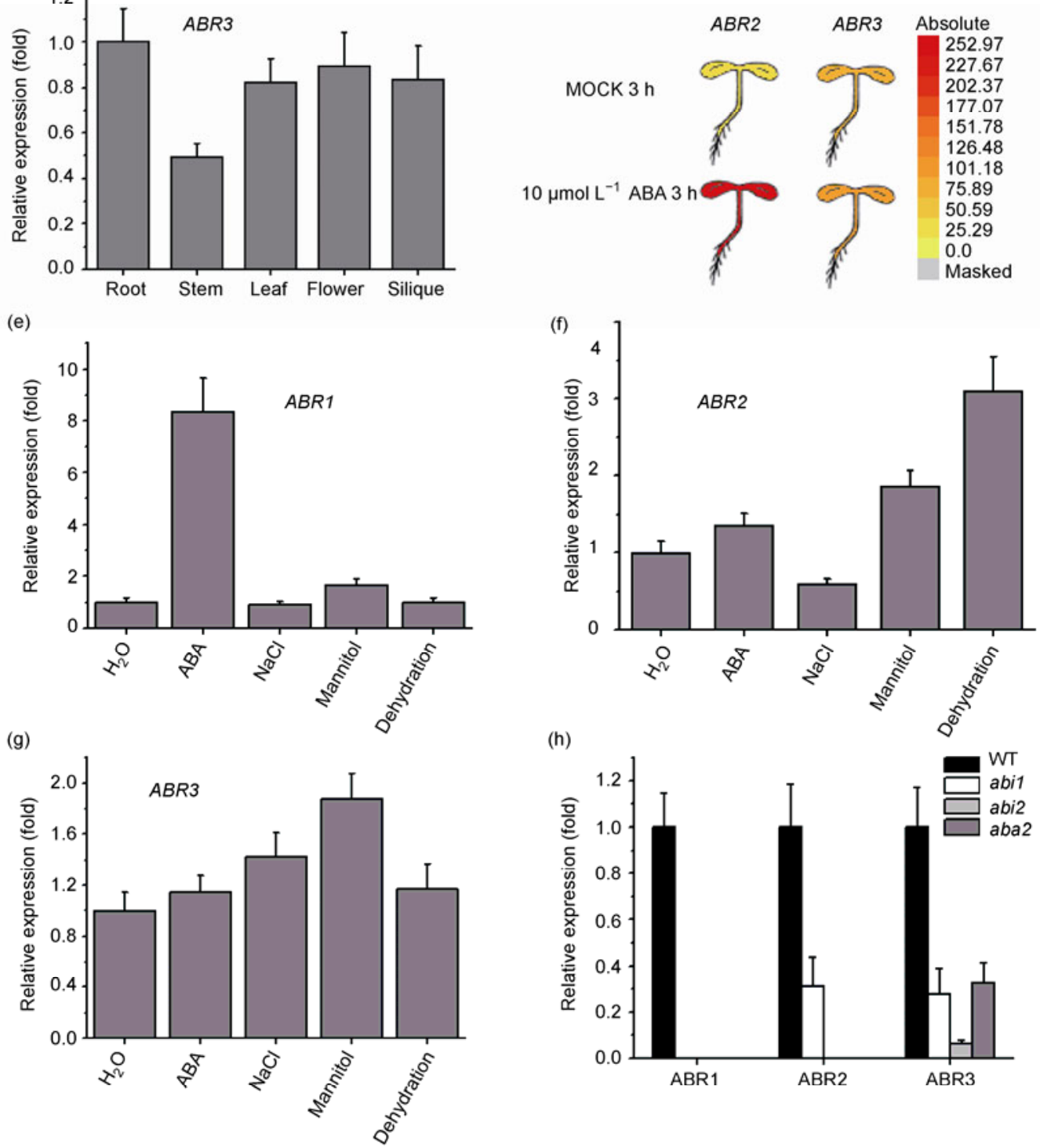

Figure 4 Analysis of $A B R 1, A B R 2$ and $A B R 3$ expressions. (a)-(c) qRT-PCR analysis of $A B R 1, A B R 2$ and $A B R 3$ expression in various tissues; (d) expression of $A B R 2$ and $A B R 3$ in the gene expression database; (e)-(g) qRT-PCR analysis of $A B R 1, A B R 2$ and $A B R 3$ expression induced by abiotic stresses; (h) qRT-PCR analysis of $A B R 1, A B R 2$ and $A B R 3$ expression in the WT and abil, abi2, aba2 mutants. 
imply that there is functional differentiation in the $A B R$ family genes, and that the different proteins perform different functions in various organs. In addition, $A B R 1, A B R 2$ and $A B R 3$ were induced by $\mathrm{ABA}$, salt, and mannitol, and dehydration stresses, particularly by ABA (Figure 4(e), (f), and $(\mathrm{g}))$. These results confirmed the results of the gene expression database (Figure 4(d)). Together, these results also suggested that $A B R 1, A B R 2$ and $A B R 3$ are involved in a variety of $A B A$-mediated stresses.

To further clarify the mechanisms of $A B R 1, A B R 2$ and $A B R 3$ responses to ABA signaling, we analyzed the expression of $A B R 1, A B R 2$ and $A B R 3$ in abil, abi2 and aba2 deletion mutants and the WT. The results show that the expression levels of $A B R 1, A B R 2$ and $A B R 3$ were significantly decreased in abil, abi2 and aba2 mutants. The expression of $A B R I$ was completely inhibited in these mutants, the expression of $A B R 2$ was completely inhibited in abi2 and $a b a 2$, and the expression of $A B R 3$ was significantly suppressed in all three mutants (Figure $4(\mathrm{~h})$ ). Differences in the expressions of $\mathrm{ABR}$ family genes in $\mathrm{ABA}$ signaling pathway-related mutants also reflect differences in the function of these genes in response to stress signals.

\section{Discussion}

The abrl/2/3 triple mutant is insensitive to ABA during seed germination and post-germination; moreover, the expressions of $A B R 1, A B R 2$ and $A B R 3$ are remarkably suppressed in abil and abi2 deletion mutants. These results clearly show that the ABR family genes are involved in ABA-regulated abiotic stress responses in plants. Barley aba45 was first protein encoding a GRAM domain to be identified in plants. The aba45 gene, which is mainly expressed in the developing aleurone layer, is induced by ABA [13]. In pepper, $A B R I$ is induced by $\mathrm{ABA}$ and encodes a protein containing only one GRAM domain, which is involved in the regulation of ABA signaling, and infection and defense responses as a negative regulator [31]. Therefore, we believe that the ABR family is an important component of the ABA signaling pathway, which is supported by genetic analysis in Arabidopsis.

The GRAM domain is considered a characteristic domain of ABA-responsive proteins. Another member of the family, tobacco ACRE140, is rapidly activated by Avr9/cf-9 [32]. In rice, Osnop encodes a C2-GRAM domain protein that causes a no pollen phenotype when the gene is deleted [33]. In Arabidopsis, VADI encodes a GRAM domain-containing protein that is involved in virus infection, defense responses, and apoptosis of vascular tissue [34]. Another gene, ROR-1 (At3g59660), is involved in the metabolism of active cytokinins, and ABA content of ror-1 is significantly lower than that of the WT [35]. Studies have shown that ABR3 (GEM) is involved in cell division, and pattern and differentiation of the root epidermal cells in Arabidopsis
[36,37]. In addition, interaction between ABR3 (GEM) and PRSL1 (At4g40100) plays a role as a regulatory subunit of PP1 [38]. Taken together, these studies illustrate that the GRAM domain-containing ABR family proteins may be involved in biotic and abiotic stress responses in plants.

In this study, we analyzed the biological functions of ABA-responsive protein encoded by $A B R 1, A B R 2$, and $A B R 3$. Bioinformatic analysis showed that $A B R 1, A B R 2$ and $A B R 3$ contain multiple ABRE cis-acting elements and that the expression of these genes can be induced by ABA. Previous studies showed that many $A B R$ family genes responded to various environmental factors $[13,20]$, which supports our view. ABR1, ABR2 and ABR3 respectively contain a GRAM domain that is considered to play an important role in the metabolic process of attachment to membranes [15]. However, most of the functions of ABR proteins are unclear. In mice, nematodes and yeast, ABR proteins contain 20 other types of domains in addition to the GRAM domain; however, most ABR proteins in Arabidopsis contain only one GRAM domain, and no other known motif or domain, which is similar in rice. The corresponding sequence analysis also suggested that the ABR protein family of rice and Arabidopsis differentiated from a common ancestor [20].

We initially predicted that $A B R 1, A B R 2$ and $A B R 3$ may be located on the plasma membrane, because the GRAM domain is considered to be associated with membrane attachment [15]; however, Arabidopsis mesophyll protoplasts transient expression experiments showed that only ABR1 is localized in the endomembrane system; ABR2 and ABR3 are located in the cytoplasm. Therefore, we conclude that the function of the GRAM domain of ABR1, ABR2 and ABR3 may depend on organelle membranes.

However, as the functional domain of ABA-responsive proteins, the most important function of the GRAM domain is in the response to ABA-regulated abiotic stress. In this paper, although $a b r l, a b r 2$ and $a b r 3$ single mutants did not show any abnormal phenotype, seed germination of the double mutants and the triple mutant were insensitive to ABA, $\mathrm{NaCl}$, mannitol and glucose. qRT-PCR analysis showed that $A B R 1, A B R 2$ and $A B R 3$ gene were expressed in various plant tissues. $A B R 1$ and $A B R 2$ had similar expression patterns, especially in leaves. These results may suggest that $A B R 1$ and $A B R 2$ have a similar regulation pattern; however, the subcellular localizations of ABR1 and ABR2 are significantly different. Therefore, the difference in gene expression patterns and protein subcellular localizations indicate that the functional differentiation of ABR1, ABR2 and ABR3 are in response to stresses. qRT- PCR analysis indicated that the expressions of $A B R 1, A B R 2$, and $A B R 3$ were upregulated to different degrees under ABA treatment, but were significantly suppressed in abil, abi2 and $a b a 2$ deletion mutants. ABA2 is a key enzyme in the ABA biosynthesis pathway, and $\mathrm{ABA}$ synthesis is defective in the $a b a 2$ deletion mutant. Seed germination and seedling 
growth of the aba2 mutant showed decreased sensitivity to mannitol, sorbitol, salt and glucose [39]. ABI1 and ABI2 are direct downstream components of the ABA receptor in the ABA signaling pathway, and abil and abi2 deletion mutants also showed insensitivity to ABA, salt, and osmotic stress and inhibited seed germination, and are located in the nucleus and cytoplasm [40-44]. Therefore, that the expressions of $A B R 1, A B R 2$ and $A B R 3$ and the phenotypes of the deletion mutants were similar suggested that $A B R 1, A B R 2$ and $A B R 3$ are involved in ABA-regulated stress responses under the regulation of $A B I 1$ and $A B I 2$. Whether ABA signaling alters the protein level of ABR1, ABR2 and ABR3, whether they respond to the ABA signal together with other family proteins, and how they regulate downstream signaling require further study.

This work was supported by the National Natural Science Foundation of China (91117002)

1 Cutler S R, Rodriguez P L, Finkelstein R R, et al. Abscisic acid: Emergence of a core signaling network. Annu Rev Plant Biol, 2010, 61: 651-679

2 Gosti F, Beaudoin N, Serizet C, et al. ABI1 protein phosphatase $2 \mathrm{C}$ is a negative regulator of abscisic acid signaling. Plant Cell, 1999, 11: 1897-1910

3 Fujii $\mathrm{H}$, Chinnusamy V, Rodrigues A, et al. In vitro reconstitution of an abscisic acid signalling pathway. Nature, 2009, 462: 660-664

4 Ma Y, Szostkiewicz I, Korte A, et al. Regulators of PP2C phosphatase activity function as abscisic acid sensors. Science, 2009, 324: 1064-1068

5 Park S Y, Hiroaki F, Yang Z, et al. Abscisic acid inhibits type 2C protein phosphatases via the PYR/PYL family of START proteins. Science, 2009, 324: 1068-1071

$6 \mathrm{He}$ Y, Gan S. A novel zinc-finger protein with a proline-rich domain mediates ABA-regulated seed dormancy in Arabidopsis. Plant Mol Biol, 2004, 54: 1-9

7 Giraudat J, Hauge B M, Valon C, et al. Isolation of the Arabidopsis ABI3 gene by positional cloning. Plant Cell, 1992, 4: 1251-1261

8 Van Z M, Carles A, Li Y, et al. Control and consequences of chromatin compaction during seed maturation in Arabidopsis thaliana. Plant Signal Behav, 2012, 7: 338-341

9 Park J, Lee N, Kim W, et al. ABI3 and PIL5 collaboratively activate the expression of SOMNUS by directly binding to its promoter in imbibed Arabidopsis seeds. Plant Cell, 2011, 23: 1404-1415

10 Finkelstein R, Gampala S, Rock C. Abscisic acid signaling in seeds and seedlings. Plant Cell, 2002, 14(Suppl): S15-S45

11 Schmitz N, Abrams S R, Kermode A R. Changes in ABA turnover and sensitivity that accompany dormancy termination of yellow-cedar (Chamaeyparis nootkatensis) seeds. J Exp Bot, 2002, 53: 89-91

12 Xiong L, Gong Z, Rock C D. Modulation of abscisic acid signal transduction and biosynthesis by a Sm-like protein in Arabidopsis. Dev Cell, 2001, 1: 771-781

13 Liu J H, Luo M, Cheng K J, et al. Identification and characterization of a novel barley gene that is ABA-inducible and expressed specifically in embryo and aleurone. J Exp Bot, 1999, 50: 727-728

14 Banno H, Chua N H. Characterization of the Arabidopsis formin-like protein AFH1 and its interacting protein. Plant Cell Physiol, 2000, 41: 617-626

15 Doerks T, Strauss M, Brendel M, et al. GRAM, a novel domain in glucosyltransferases, myotubularins and other putative membraneassociated proteins. Trends Biochem Sci, 2000, 25: 483-485

16 Berger P, Schaffitzel C, Berger I, et al. Membrane association of myotubularin-related protein 2 is mediated by a pleckstrin homology-
GRAM domain and a coiled-coil dimerization module. Proc Natl Acad Sci USA, 2003, 100: 12177-12182

17 Choudhury P, Srivastava S, Li Z, et al. Specificity of the myotubularin family of phosphatidylinositol-3-phosphatase is determined by the PH/GRAM Domain. J Biol Chem, 2006, 281: 31762-31769

18 Tsujita K, Itoh T, Ijuin T, et al. Myotubularin regulates the function of the late endosome through the GRAM domain-phosphatidylinositol 3,5-bisphosphate interaction. J Biol Chem, 2004, 279: 1381713824

19 Masahide O, Dirk W, Takeshi N, et al. Peroxisome degradation requires catalytically active sterol glucosyltransferase with a GRAM domain. EMBO J, 2003, 22: 3231-3141

20 Shu Y J, Rengasamy R, Srinivasan R. Comparative transcriptional profiling and evolutionary analysis of the GRAM domain family in eukaryotes. Dev Biol, 2008, 314: 418-432

21 Yoo S D, Cho Y H, Sheen J. Arabidopsis mesophyll protoplasts: A versatile cell system for transient gene expression analysis. Nat Protoc, 2007, 2: 1565-1572

22 Nakashima K, Fujita Y, Katsura K, et al. Transcriptional regulation of ABI3- and ABA-responsive genes including RD29B and RD29A in seeds, germinating embryos, and seedlings of Arabidopsis. Plant Mol Biol, 2006, 60: 51-68

23 Kaplan B, Davydov O, Knight H, et al. Rapid transcriptome changes induced by cytosolic $\mathrm{Ca}^{2+}$ transients reveal ABRE-related sequences as $\mathrm{Ca}^{2+}$-responsive cis elements in Arabidopsis. Plant Cell, 2006, 18: 2733-2748

24 Choi H, Hong J, Ha J, et al. ABFs, a family of ABA-responsive element binding factors. J Biol Chem, 2000, 275: 1723-1730

25 Kang J Y, Choi H I, Im M Y, et al. Arabidopsis basic leucine zipper proteins that mediate stress-responsive abscisic acid signaling. Plant Cell, 2002, 14: 343-357

26 Oh S J, Song S I, Kim Y S, et al. Arabidopsis CBF3/DREB1A and $\mathrm{ABF} 3$ in transgenic rice increased tolerance to abiotic stress without stunting growth. Plant Physiol, 2005, 138: 341-351

27 Choi H I, Park H J, Park J H, et al. Arabidopsis calcium-dependent protein kinase AtCPK32 interacts with $\mathrm{ABF} 4$, a transcriptional regulator of abscisic acid-responsive gene expression, and modulates its activity. Plant Physiol, 2005, 139: 1750-1761

28 Yamaguchi S K, Shinozaki K. Identification of a cis-regulatory region of a gene in Arabidopsis thaliana whose induction by dehydration is mediated by abscisic acid and requires protein synthesis. Mol Gen Genet, 1995, 247: 391-398

29 Luo M, Wang Y Y, Liu X, et al. HD2C interacts with HDA6 and is involved in ABA and salt stress response in Arabidopsis. J Exp Bot, 2012, 63: 3297-3306

30 Gao G, Zhang S, Wang C, et al. Arabidopsis CPR5 independently regulates seed germination and postgermination arrest of development through LOX pathway and ABA signaling. PLoS One, 2011, 6: e19406

31 Du S C, Byung K H. Proteomics and functional analyses of pepper abscisic acid-responsive 1 (ABR1), which is involved in cell death and defense signaling. Plant Cell, 2011, 23: 823-842

32 Rowland O, Ludwig A A, Merrick C J, et al. Functional analysis of Avr9/Cf-9 Rapidly elicited genes identifies a protein kinase, ACIK1, that is essential for full Cf-9-dependent disease resistance in tomato. Plant Cell, 2005, 17: 295-310

33 Jiang S Y, Cai M, Ramachandran S. The Oryza sativa no pollen (Osnop) gene plays a role in male gametophyte development and most likely encodes a C2-GRAM domain-containing protein. Plant Mol Biol, 2005, 57: 835-853

34 Severine L, Bai q L, Marie C A, et al. VASCULAR ASSOCIATED DEATH1, a novel GRAM domain-containing protein, is a regulator of cell death and defense responses in vascular tissues. Plant Cell, 2004, 16: 2217-2232

35 Somya D, Radomira V, Vaclav M, et al. Characterization of Arabidopsis thaliana mutant ror-1 (roscovitine-resistant) and its utilization in understanding of the role of cytokinin $\mathrm{N}$-glucosylation pathway in plants. Plant Growth Regul, 2010, 61: 231-242

36 Caro E, Castellano M M, Gutierrez C. A chromatin link that couples 
cell division to root epidermis patterning in Arabidopsis. Nature, 2007, 447: 213-217

37 Elena C M, Mar C, Crisanto G. GEM, a novel factor in the coordination of cell division to cell fate decisions in the Arabidopsis epidermis. Plant Signal Behav, 2007, 2: 494-495

38 Takemiya A, Yamauchi S, Yano T, et al. Identification of a regulatory subunit of protein phosphatase 1 which mediates blue light signaling for stomatal opening. Plant Cell Physiol, 2012, 54: 24-35

39 Gonzalez G M, Apostolova N, Belles J M, et al. The short-chain alcohol dehydrogenase ABA2 catalyzes the conversion of xanthoxin to abscisic aldehyde. Plant Cell, 2002, 14: 1833-1846

40 Lin P C, Hwang S G, Endo A, et al. Ectopic expression of ABSCISIC ACID 2/GLUCOSE INSENSITIVE 1 in Arabidopsis promotes seed dormancy and stress tolerance. Plant Physiol, 2007, 143: 745-758
41 Leung J, Merlot S, Giraudat J. The Arabidopsis ABSCISIC ACIDINSENSITIVE2 (ABI2) and ABI1 genes encode homologous protein phosphatases $2 \mathrm{C}$ involved in abscisic acid signal transduction. Plant Cell, 1997, 9: 759-771

42 Stefan H, Michele M, Juan P S, et al. Genome-wide gene expression profiling in Arabidopsis thaliana reveals new targets of abscisic acid and largely impaired gene regulation in the abi1-1 mutant. J Cell Sci, 2002, 115: 4891-4900

43 Hai L S, Xiao J W, Wei H D, et al. Identification of an important site for function of the type $2 \mathrm{C}$ protein phosphatase ABI2 in abscisic acid signalling in Arabidopsis. J Exp Bot, 2011, 62: 5713-5725

44 Wan H C, Akira E, Li Z, et al. A unique short-chain dehydrogenase/reductase in Arabidopsis glucose signaling and abscisic acid biosynthesis and functions. Plant Cell, 2002, 14: 2723-2743

Open Access This article is distributed under the terms of the Creative Commons Attribution License which permits any use, distribution, and reproduction in any medium, provided the original author(s) and source are credited. 